\title{
Persistent Aneurysm Growth Following Pipeline Embolization Device Assisted Coiling of a Fusiform Vertebral Artery Aneurysm - A Word of Caution!
}

\author{
Mena Kerolus, MD², Manish K. Kasliwal, MD, MCh, Demetrius K. Lopes, MD
}

The complex morphology of vertebrobasilar fusiform aneurysms makes them one of the most challenging lesions treated by neurointerventionists. Different management strategies in the past included parent vessel occlusion with or without extra-intracranial bypass surgery and endovascular reconstruction by conventional stents. Use of flow diversion has emerged as a promising alternative option with various studies documenting its efficacy and safety. However, there are various caveats associated with use of flow diversion in patients with fusiform vertibrobasilar aneurysms especially in patients presenting with acute subarachnoid hemorrhage $(\mathrm{SAH})$. We report a rare case of persistent aneurysmal growth after coiling and placement of the Pipeline Embolization Device (PED; ev3, Irvine, California, USA) for SAH from a fusiform vertebral artery aneurysm. As consequences of aneurysm rupture can be devastating especially in patients with a prior $\mathrm{SAH}$, the clinical relevance of recognizing and understanding such patterns of failure cannot be overemphasized as highlighted in the present case.

Key Words : Aneurysm; Complication; Flow Diverter; Pipeline Embolization Device; Recurrence; Subarachnoid Hemorrhage; Treatment

Vertebrobasilar fusiform aneurysm remains one of the most formidable lesions treated by neurointerventionists because there has been no well accepted management paradigm. Various modes of presentation may include ischemic stroke, cranial nerve palsies,

\footnotetext{
All authors: Department of Neurosurgery, Rush University Medical Center, Chicago

Received September 23, 2014;

accepted after revision December 17, 2014

Correspondence to: Manish K. Kasliwal, MD, MCh, Department of Neurosurgery, 1725 W Harrison Street, Suite 855, Rush University Medical Center, Chicago, IL-60612, U.S.A.

Tel. 312.942.6644 Fax. 312.942.2176

E-mail: Manish_Kasliwal@rush.edu

This is an Open Access article distributed under the terms of the Creative Commons Attribution Non-Commercial License (http://creativecommons.org/licenses/by-nc/3.0) which permits unrestricted non-commercial use, distribution, and reproduction in any medium, provided the original work is properly cited.
}

brainstem compression, hydrocephalus, intracranial hemorrhage and subarachnoid hemorrhage (SAH) [1]. The complex morphology of fusiform aneurysms makes both surgical as well as endovascular treatment challenging. Different management strategies include parent vessel occlusion with or without extra-intracranial bypass surgery and endovascular reconstruction by conventional stents $[1,2]$. The recent development and food and drug administration (FDA) approval of flowdiverting stents such as the Pipeline Embolization Device (PED; ev3, Irvine, California, USA) has dramatically increased the applicability of endovascular treatment options for complex cerebral aneurysms that were previously considered unfavorable or contraindications for endovascular treatment [3]. While currently FDA approved for large or giant necked aneurysms involving the internal carotid artery (ICA) from the petrous to the superior hypophyseal segments, as 
experience continues to grow with use of this new device, there has been a trend towards use of flow diversion even in patients with SAH either with or without coil assistance, in aneurysms beyond the level of circle of Willis and fusiform vertebrobasilar trunk aneurysms $[4,5]$. While the use of PED alone may be optimal in patients with pseudoaneurysm, blister aneurysm, or dissecting-type aneurysm in the absence of SAH, use of stent alone without coiling does not provided immediate protection and is not ideal for aneurysm presenting with $\mathrm{SAH}$ as there remains the risk of re-rupture which can be fatal. Chung, et al, recently reported the use of stent-assisted coiling in patients with SAH and showed increased rate of complications with this type of approach.6 Interestingly, most of the complications were related to the bleeding complications secondary to use of anti-platelet agents especially during placement of external ventricular drain (EVD) and shunts. As there is always a significant enthusiasm in adoption of newer technologies, recognition of patterns of complications and mechanisms of failure remains paramount to optimize the appropriate use of these devices and prevention of complications. Excellent occlusion rates have been reported with the use of PED in the treatment of various intracranial aneurysms. Even though the occlusion rate following PED embolization is not $100 \%$, persistent aneurysm growth following the use of a PED is very unusual. We report this rare case of persistent aneurysmal growth after coiling and placement of a PED for SAH from a fusiform vertebral artery aneurysm with discussion of various nuances associated with the use of PED in patients with fusiform aneurysms.

\section{CASE REPORT}

A 43-year-old male with a history of uncontrolled hypertension was admitted to our neuroscience intensive care unit form an outside institution with a diagnosis of a Hunt \& Hess grade 4/Fisher grade 3 SAH (Fig. 1A). An EVD was placed on admission and a digital subtraction angiography (DSA) was performed which demonstrated presence of the right vertebral artery fusiform aneurysm with the posterior inferior cerebellar artery (PICA) arising from the base of a focal dilatation from the fusiform aneurysm segment (Fig. 1B). After consideration of all the treatment options, endovascular treatment was elected in view of poor grade SAH and patients' cardiopulmonary status. He underwent coil embolization along with placement of a PED across the aneurysm. A 6-French guide catheter was used to selectively catheterize the right vertebral artery. A microcatheter was subsequently placed into the vertebral artery with coiling of the focal dilatation arising off the aneurysm along with deployment of a $3.75 \times 18 \mathrm{~mm}$ PED completely covering the fusiform vertebral aneurysm for vessel remodeling. A Raymond class II occlusion of the focal dilatation arising from the fusiform aneurysm was achieved (Fig. 1C). The patient was loaded with aspirin and clopidogrel during the procedure. The EVD was converted to a ventriculo-peritoneal shunt before the patient was discharged home in a good neurological condition. $\mathrm{He}$ was alert and oriented with no focal deficits at discharge except presence of right sided sixth cranial nerve palsy. Dual antiplatelet agents were prescribed for 6 months following the procedure. Routine follow up angiography done at 6 months as per our institutional protocol showed coil compaction and enlargement and regrowth of both the focal dilatation and the fusiform aneurysm without migration of the pipeline stent (Fig. 1D). Even though the patient was asymptomatic, considering significant enlargement of the aneurysm with a prior presentation with $\mathrm{SAH}$, he was taken back to the neuro-interventional suite with placement of three overlapping PED's $(3.5 \mathrm{~mm} \times 18 \times 2$ and $3.5 \times 14 \mathrm{~mm}$ ) to increase the overall coverage area and further reduce the shear stress which possibly caused the aneurysm to continue growing despite treatment with a single PED on presentation. The post procedure course was uneventful and he was discharged home with no new neurological deficits. A follow up angiography was performed at 3 months which showed no residual aneurysm (Fig. 1E) with no neurological deficits on clinical examination.

\section{DISCUSSION}

Fusiform aneurysms represent a challenging subgroup of intracranial aneurysms which generally presents with ischemic stroke, cranial nerve palsies, brainstem compression, hydrocephalus and intracranial hemorrhage [1]. SAH is generally uncommon in patients with fusiform aneurysm; but when present increases the complexity associated with management of these aneurysms [1]. Given the complex morphology of fusiform aneurysms, both endovascular and surgical treatment options remain limited and technically challenging [1].

Poor grade SAH along with associated co-morbid medical conditions often results in pursuing the 


\section{Mena Kerolus, et al.}

endovascular route for treatment of intracranial aneurysms even for cases for which there is potential for increased complications [6]. Use of stents in ruptured $\mathrm{SAH}$ is such an indication as they often require administration of dual antiplatelet agents which associated risks of hemorrhagic complications, either
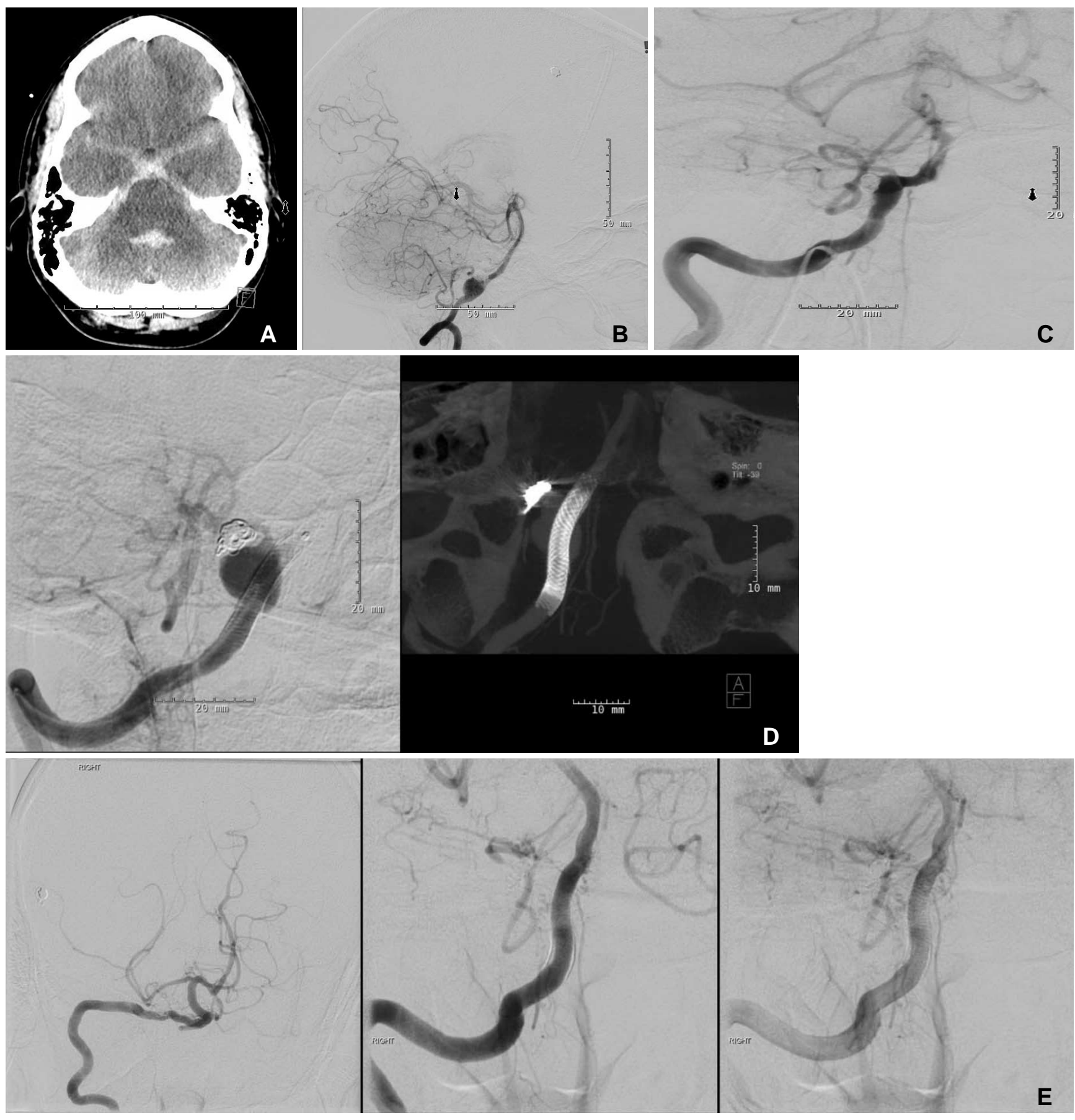

D

Fig. 1. A. Axial computed tomography (CT) scan of the head showing presence of Fisher grade $3 \mathrm{SAH}$.

B. Digital subtraction angiography (DSA) demonstrating right vertebral artery fusiform aneurysm with the PICA takeoff arising from the base of a focal dilatation from the fusiform aneurysm segment.

C. Post embolization DSA showing deployment of a $3.75 \times 18 \mathrm{~mm}$ PED completely covering the fusiform vertebral aneurysm along with Raymond class II occlusion of the focal dilatation arising from the fusiform aneurysm.

D. Follow up angiogram at 6 months showing the presence of aneurysm regrowth and coil compaction (left panel) despite well deployed PED across the aneurysm (right panel).

E. Anteroposterior (left panel) and lateral (middle and right panels) images from DSA performed 3 months after the second procedure showing remodeling of the right vertebral artery with no residual or recurrent aneurysm. 
spontaneous or associated with interventions such as placement or revision of EVDs and VPS [6]. Flow diverters induce disruption of flow near the aneurysm neck, inducing thrombosis into the aneurysm sac while preserving physiological flow in the parent vessel and adjacent branches and have been the most recent advancement in endovascular treatment of intracranial aneurysms [3]. The term "flow diversion" refers to the concept of hemodynamic influence on an aneurysm by reducing both inflow and outflow with flow diverters being stent-like endovascular implants designed to alter the blood flow in an artery in a way that favors the flow along the longitudinal axis of the target vessel and reduces inflow and outflow of an aneurysm covered by the device [3]. Various flow diverters commercially available includes CE mark device (Silk, Balt Extrusion, Montmorency, France and the Pipeline Embolization Device (PED, ev3, Irvine, CA, USA). A tight mesh of small cell sizes and a high longitudinal flexibility of these newer flow diverters offer the possibility to cover an aneurysm orifice and in carefully selected cases, may successfully be used to reconstruct intracranial vessel dissections [3].

While approved for large or giant necked aneurysms involving the ICA from the petrous to the superior hypophyseal segments, a number of studies have shown the effectiveness of stent-assisted coiling in SAH with good overall clinical outcome; albeit at higher rates of complications [6, 7]. Most of these complications have been hemorrhagic secondary to the use of antiplatelet and anticoagulant medications [6]. However, once the acute period is over with good aneurysm occlusion, delayed thrombosis of the stent remains the foremost concern along with other device-related complications such as creeping of the stent or migration/shortening of the PED [8].

Overall, the use of PED has been shown to result in excellent aneurysm occlusion in various series. While immediate angiographic exclusion of the aneurysm at the end of the original procedure is observed only in up to $10-20 \%$ of patients treated with PED, total aneurysm occlusion rates of more than $90 \%$ at 1 year have been reported in various studies even in large and giant aneurysms and even fusiform and dissecting type aneurysms through remodeling of the parent vessel over time. The use of PED in wide neck aneurysms makes it an ideal choice to be used in conjunction with coil placement as conventional stent porosity would not provide significant protection against coil migration as compared to PED in some cases where smaller coils needs to deployed [9]. While the lack of immediate aneurysm occlusion with flow diverters necessitates obliteration of aneurysm sac using simultaneous coil placement in patients with $\mathrm{SAH}$, as the occlusion rates with use of flow diverting stents such as PED increases up to $90 \%$ at one year follow up, any residual aneurysm neck after securing of the aneurysm dome should not be concerning as the flow diverter stent should actually lead to complete aneurysm occlusion due to its mechanism of action [9].

The case described in this report was treated with this hypothesis in mind. To avoid the possibility of coil migration/herniation without a scaffold, a PED was used to address this and also to treat the fusiform vertebral artery aneurysm. The use of conventional low coverage stent assisted coiling was not considered optimal due to the origin of the PICA from the fusiform aneurysm segment of the artery. Placement of flow diverter PED was considered the best option as it would allow coiling of the focal dilatation of the aneurysm and leave the PICA patent due to maintenance of the flow secondary to associated pressure gradient. Hence once positioned and prior to coil detachment into the focal dilatation from the aneurysm, a $3.75 \mathrm{~mm} \times 18 \mathrm{~mm}$ PED device was partially deployed to cover the PICA and the fusiform aneurysm while temporarily jailing the microcatheter. The coil was then detached, and the remainder of the stent was deployed, preventing migration of the coil mass. The combined use of both modalities, the coil and the PED, provided immediate protection from re-hemorrhage, while counting on the long term protection through remodeling of the parent vessel by the PED.

Unfortunately, in our case, the follow up angiogram showed aneurysm regrowth with coil compaction despite well positioned PED which required another procedure. The plausible hypothesis for aneurysm expansion in our case may be related to the low coverage of the fusiform segment of the aneurysm which allowed progression of the aneurysm in the diseased fusiform segment due to persistent shear stress in the vessel and led to recurrence of the aneurysm. We hypothesize that in such cases it may be important to reduce the shear stress in the vessel wall to allow vessel remodeling; failure of which may result in persistent growth of the fusiform aneurysm as was seen in the present case. Unfortunately, the number of PED to be employed and what is constituted as adequate coverage is considered controversial with no consensus. Technically, a single PED is considered adequate if the target lesion is fully covered and in case of immediate contrast medium stagnation. However, placement of 


\section{Mena Kerolus, et al.}

multiple devices may be of value in cases with an unchanged aneurysm perfusion after implantation of PED indicating an insufficient effect of flow diversion and cases of widespread longitudinal vessel dilatation where a telescoping implantation of multiple devices was necessary to cover the entire lesion.

While the use of multiple PEDs appears intuitive in cases with fusiform aneurysms to decrease the shear stress on the diseased vessel wall, it may not obviate the chances of incomplete occlusion in these subgroup as seen in the study by Jabbour et al. [10] and may unnecessarily increase procedural time, procedural cost, and the risk of thromboembolic complications [2]. On the other hand, a dense packing of the device during the implantation process does raise the degree of coverage resulting in larger flow diversion and a larger protective effect arguing in favor of implantation of more than one flow diverter across the orifice in order to increase the density with an improved flow diversion towards the parent artery. The benefit of increased density needs to be balanced with risk of side branch or parent vessel occlusions due to an in-stent thrombosis and other thromboembolic complications [2]. We believe that in the absence of definitive evidence for either and balancing the risk benefits ratio, concept of a staged therapy with initial rather defensive implantation of devices followed by additional flow diverters dependent on the follow-up results appears intuitive. While this approach may be useful for patients who does not presents with SAH, poor response with aneurysm regrowth in patients with SAH may be concerning due to risk of re-rupture as could have happened in our case. In these cases of fusiform aneurysm presenting with SAH, it may be prudent to utilize multiple PEDs to increase the coverage area; what is considered adequate is debatable and is up to the discretion of the treating neurointerventionlist as even with more than one PED, Jabbour et al. [10] reported with poor outcome in patients with fusiform aneurysm. Hence considering an earlier follow up angiography in patients with fusiform aneurysms treated with PED especially in patients with SAH may be of value to detect increase in size of the aneurysm earlier and tailor the number of devices necessary to achieve the desired outcome or pursue alternative treatment modality to prevent aneurysm rupture.

Fischer, et al recently reported complete aneurysm occlusion or decrease in size of the aneurysm in about $96 \%$ of patients with fusiform or dissecting aneurysm at a follow up of 1 year [11]. Interestingly, in the same study; only $24 \%$ of cases showed complete cure and
$30 \%$ showed partial occlusion at 3 months follow-up angiography. There was no aneurysm growth in the study at three or one year follow-up angiogram demonstrating the safety and efficacy of PED in fusiform and dissecting aneurysms [11]. None of the patients in the study had acute SAH though, and a number of patients were treated with more than one PEDs. In contrast to this study, Siddiqui et al reported less favorable results in a series of 7 large or giant vertebrobasilar fusiform aneurysms treated with flow diverters with significant morbidity and mortality; the poor outcome being secondary to post-treatment aneurysm ruptures in two and lack of neurological improvement caused by brainstem infarcts in another 2 patients [12]. Suboptimal outcome with the use of PED in fusiform aneurysms was also echoed in the study by Jabbour, et al in which they found fusiform aneurysm shape as a negative predictor for aneurysm obliteration despite a significantly higher number of PEDs (1.8) used in this subset of patients [10].The variable results from different studies highlights the complexity associated with use of PED in vertebrobasilar fusiform aneurysms. As the data on the use of PED in vertebrobasilar aneurysms presenting with SAH is not robust, clinicians should be careful in extrapolating the excellent outcome following the use of flow diverters in well studied conditions. Nevertheless, with the increasing use of PED outside more common FDA approval indications [3], apart from demonstrating the efficacy, the clinical relevance of understanding the patterns of failure cannot be overemphasized as highlighted in the present case.

In conclusion, an unusual case of persistent aneurysmal growth following treatment with PED assisted coiling of a fusiform vertebral artery aneurysm is reported. As the use of PED is becoming increasingly common for a number of clinical indications, the mechanism and instances of failure needs to be understood to prevent complications and optimize the use of this newer device. We suggest considering an earlier follow up angiography in patients with fusiform aneurysms treated with PED to detect inadequate response to treatment with possible need of subsequent multiple/overlapping PED to increase the chances of successful outcome.

Disclosure: Dr. Lopes has financial and research relationships with Penumbra, Covidien, and Stryker. Other authors have no disclosures, 


\section{Pipeline Embolization Device for Fusiform Aneurysm}

\section{References}

1. Wang Q, Leng B, Song D, Chen G. Fusiform aneurysms of the vertebrobasilar arterial trunk: choice of endovascular methods and therapeutic efficacy. Acta Neurochir (Wien) 2010;152:1467-1476

2. Tan LA, Keigher KM, Munich SA, Moftakhar R, Lopes DK. Thromboembolic complications with Pipeline Embolization Device placement: impact of procedure time, number of stents and pre-procedure P2Y12 reaction unit (PRU) value. J Neurointerv Surg 2015;7:217-221

3. Becske T, Kallmes DF, Saatci I, McDougall CG, Szikora I, Lanzino $\mathrm{G}$, et al. Pipeline for uncoilable or failed aneurysms: results from a multicenter clinical trial. Radiology 2013;267:858868

4. Cruz JP, O’Kelly C, Kelly M, Wong JH, Alshaya W, Martin A, et al. Pipeline embolization device in aneurysmal subarachnoid hemorrhage. AJNR Am J Neuroradiol 2013;34:271-276

5. Pistocchi S, Blanc R, Bartolini B, Piotin M. Flow diverters at and beyond the level of the circle of willis for the treatment of intracranial aneurysms. Stroke 2012;43:1032-1038

6. Chung J, Lim YC, Suh SH, Shim YS, Kim YB, Joo JY, et al. Stent-assisted coil embolization of ruptured wide-necked aneurysms in the acute period: incidence of and risk factors for periprocedural complications. J Neurosurg 2014;121:4-11
7. Martin AR, Cruz JP, Matouk CC, Spears J, Marotta TR. The pipeline flow-diverting stent for exclusion of ruptured intracranial aneurysms with difficult morphologies. Neurosurg 2012;70(1 Suppl Operative):21-28

8. Chalouhi N, Tjoumakaris SI, Gonzalez LF, Hasan D, Pema PJ, Gould G, et al. Spontaneous delayed migration/shortening of the pipeline embolization device: report of 5 cases. AJNR Am J Neuroradiol 2013;34:2326-2330

9. Lylyk P, Miranda C, Ceratto R, Ferrario A, Scrivano E, Luna HR, et al. Curative endovascular reconstruction of cerebral aneurysms with the pipeline embolization device: the Buenos Aires experience. Neurosurgery 2009;64:632-642

10. Jabbour P, Chalouhi N, Tjoumakaris S, Gonzalez LF, Dumont AS, Randazzo C, et al. The Pipeline Embolization Device: learning curve and predictors of complications and aneurysm obliteration. Neurosurgery 2013;73:113-120

11. Fischer S, Perez MA, Kurre W, Albes G, Bazner H, Henkes H. Pipeline embolization device for the treatment of intra- and extracranial fusiform and dissecting aneurysms: initial experience and long-term follow-up. Neurosurgery 2014;75:364-374

12. Siddiqui AH, Abla AA, Kan P, Dumont TM, Jahshan S, Britz $\mathrm{GW}$, et al. Panacea or problem: flow diverters in the treatment of symptomatic large or giant fusiform vertebrobasilar aneurysms. $J$ Neurosurg 2012;116:1258-1266 